\title{
The acquisition of positive secondary reinforcement in human subjects
}

SAUL M. LEVIN AND KATHLEEN STERNER

The purpose of the present study was to determine if it is possible to build reinforcing properties into a neutral stimulus and to use the stimulus to influence verbal behavior in a different task. A pencil tap was paired with "right" during a paired-associates learning task and was then tested for reinforcing properties on a subsequent sentence construction task. The results indicated that the tap functioned as a reinforcer on the sentence construction task.

Most studies of secondary reinforcement with humans have used increased resistance to extinction as a measure of secondary reinforcement (e.g., Fort, Myers, \& Myers, 1962; Myers \& Myers, 1962). The difficulty with such a procedure is that the increased resistance to extinction may be due to generalization effects and not to secondary reinforcement. According to theory, one would expect a secondary reinforcer to be effective in influencing a response which is different from the one present in the situation in which it originally acquired its reinforcing properties. In studies with human $\mathrm{Ss}$, there are few data bearing on the hypothesis that application of an experimentally-created secondary reinforcer can effect a new response. Girardeau (1962), using mental defectives as Ss, paired an auditory stimulus with candy in the initial phase of his procedure. In a subsequent task in which the stimulus was contingent on a bar press, there was no evidence that it had become a secondary reinforcer.

The purpose of the present study was to determine if it is possible to build reinforcing properties into a neutral stimulus and to use the stimulus to influence verbal behavior in a different task. More specifically, a pencil tap was paired with "right" during a pairedassociates learning task and was then tested for reinforcing properties on a subsequent sentence construction task.

\section{Method}

Subjects. The Ss were 30 open ward psychiatric patients none of whom was markedly disturbed at the time of the experiment. The patients were assigned to an Experimental and a Control group of 15 Ss each. The Ss in the two groups were individually matched for sex, intelligence and diagnosis.

Procedure. The experimental procedure consisted of two stages-an initial paired-associates learning task and a subsequent sentence construction task.

During the paired-associates task all ss learned a 12-item list consisting of six easy pairs and six difficult pairs. On each trial the $E$ read the list of pairs aloud at the rate of one pair every $2 \mathrm{sec}$. and then presented the first word of each pair. There were four such trials. The Ss were encouraged to guess if they weren't sure of the correct answer. If the $S$ responded correctly the E said "right," while if the answers were incorrect the E said "wrong." For the Experimental group the $\mathrm{E}$ tapped the table in a casual manner with the eraser end of a pencil before saying "right." An effort was made to make the tap noticeable but not overly conspicuous.

A sentence construction task was introduced immediately after the fourth trial on the paired-associates task. The stimulus materials consisted of $1003 \times 5 \mathrm{in}$. index cards on each of which a different neutral past tense verb was typed. All of the verbs were selected from the Thorndike-Lorge list of the 1000 most frequent words in written English. The pronouns I, We You, He, She, The $y$ were typed below the verb with the order of the pronouns randomized over all the cards. The Ss were instructed to respond to each card by constructing a sentence beginning with one of the pronouns and containing the verb. From the beginning of the task the E tapped the table with a pencil immediately following every sentence beginning with $I$, or We, The Ss in both groups received the pencil tap for sentences beginning with first person pronouns. Immediately after the conditioning procedure each $\mathrm{S}$ was interviewed in an effort to determine if he or she had been aware of the contingency between the tap and $I$, $W e$, sentences. The interview consisted of 12 questions. The most direct question was "Did you ever have the idea that the tap only occurred when you chose a certain word to begin your sentences with? If so, which word?" Results

It will be recalled that during the initial learning task for the Experimental group the pencil tap was paired with a verbal reinforcer, "right," while the Control group did not experience the tap. During the sentence construction task both groups received the tap for $I, W \in$, sentences from the beginning of the procedure. If the tap acquired reinforcing properties as a result of having been paired with the verbal reinforcer, then during the sentence construction task the Experimental group should give more I, We, sentences.

Table 1 presents the mean frequencies of $I$, We,

Table 1. Mean Frequencies of I, We Sentences for Experimental and Control Groups

\begin{tabular}{lccccc} 
& \multicolumn{5}{c}{ Trials } \\
& $1-20$ & $21-40$ & $41-60$ & $61-80$ & $81-100$ \\
\hline Experimental Group & 7.47 & 9.73 & 9.60 & 9.07 & 9.30 \\
Control Group & 6.13 & 6.93 & 6.53 & 7.00 & 6.68 \\
\hline
\end{tabular}


sentences over the 100 trials for both groups. The table suggests that the Experimental group gave more I, We, sentences over trials than did the Control group. The data were analyzed in two ways. First, a mixed analysis of variance was carried out, with groups as the between-Ss factor and trials as the within-Ss factor. The significant effect of groups $(F=5.04, p<.05)$, together with Table 1, indicates that the Experimental group gave significantly morel, We sentences than did the Control group. A second analysis was conducted to determine if the Experimental group showed a greater over-all increase inl, We sentences than did the Control group. A difference score was computed for each $\mathrm{S}$ by subtracting the number of $I$, We sentences in the first block of trials from the number of such sentences in the final block. The mean difference score of the Experimental group (2.33) was greater than the mean difference score of the Control group (.53). However, the difference between these scores did not reach an acceptable level of significance $(t=1.55, p<.10)$. Thus, while the Experimental group gave significantly more I, We sentences, it did not show a clearly significant increase in the frequency of such sentences from the beginning to the end of the task.

With respect to awareness, two of the 15 experimental Ss were able to verbalize the fact that the pencil tap had occurred following I, W $e$, sentences. The performance of the remaining 13 Ss was compared, with the performance of their matched controls by means of analysis of variance. A significant effect of groups $(F=4.36, p<.05)$ indicates that the unaware Ss gave more I, We sentences than did their matched controls.

\section{Discussion}

The results of the current study suggest that it is possible to influence verbal behavior by means of a secondary reinforcer on a task different from the one in which it originally acquired its reinforcing properties. It may be possible to use the current technique, or a similar one, to study the influence on secondary reinforcement in humans of variables which have been investigated in animal work, such as number of pairings of the secondary reinforcer with the primary reinforcer, and magnitude of primary reinforcement. There are some data in the current study bearing on the influence of number of pairings. During the pairedassociates learning task there was some variability among experimental Ss with respect to the number of correct responses and hence the number of times the tap was paired with "right." Analysis of the data indicated that there was no relationship between the number of such pairings and the effectiveness of the secondary reinforcer during the sentence construction task. However, it should be borne in mind that the variability in number of pairings was not very great. The range was 23-47, with 13 of the 15 values falling between 37 and 47 tap-"right" pairings. If the initial task were changed to permit greater control over the number of pairings, a wider range of values could be studied.

The data on awareness are interesting in the light of the recent controversy regarding learning without awareness (Eriksen, 1962). In the present study, the interview for awareness was fairly detailed and still there was a group of Ss who were apparently unaware of the correct contingency and yet showed some limited evidence of having been influenced by the secondary reinforcer. However, before it is concluded that the current findings constitute evidence for the unmediated effect of a reinforcer, it should be borne in mind that the post-experimental interview could have been somewhat more direct. The Ss could have been presented with the correct contingency in the form of a question such as "Did you ever have the idea that the tap only occurred when you began your sentence with $I$, with $W \mathfrak{E}$, or with either I or $W e$ "'A negative answer to such a question, together with evidence of a significant reinforcer effect, would have constituted somewhat stronger evidence for conditioning without awareness. Notwithstanding these considerations, the present data raise the possibility that the procedure could be employed to study the direct or unmediated effects on behavior of a reinforcing stimulus.

Finally, the data leave unanswered the question of whether or not the secondary reinforcement effect was dependent on the initial contrasting of positive reinforcement trials with negative reinforcement trials on which the pencil tap was absent. In other words, would the tap have acquired reinforcing properties if, during the paired-associates task, there had been positive reinforcement trials only? There is some evidence in the animal literature to suggest that a stimulus must first be established as a discriminative stimulus in order to function as a secondary reinforcer (McGuigan \& Crockett, 1958). However, there are few data in studies with human Ss bearing on this issue.

\section{References}

Eriksen, C. W. (Ed.). Behavior and awareness: A symposium of research and interpretation. J. Pers., 1962, 30 (Supplement). Fort, J, G., Myers, J. L., \& Myers, N. A. Secondary teinforcement in a discrimination problem. J. gen. Psychol., 1962, 66, 159-168. Girardeau, F. L. The effect of secondary reinforcement on the operant behavior of mental defectives. Amer. J. ment. Defic., 1962, 67, 441-449.

McGuigan, F. J., \& Crockett, F. Evidence that the secondary reinforcing stimulus must be discriminated. J. exp. Psychol., 1958. 55, 184-187.

Myers, N. A., \& Myers, J. L. Effects of secondary reinforcement schedules in extinction on children's responding. J. exp. Psychol., 1962, 64, 586-588. 